\title{
On the Forced Response of Harmonically and Partially Mistuned Bladed Disks. Part I: Harmonic Mistuning*
}

\author{
MARC P. MIGNOLET ${ }^{a, \dagger}$, WEI HU ${ }^{a}$ and IOAN JADIC ${ }^{b}$ \\ a Department of Mechanical and Aerospace Engineering, Arizona State University, Tempe, AZ 85287-6106, USA, \\ ${ }^{b}$ Raytheon Aircraft Company, P.O. Box 85, Wichita, KS 67201-0085, USA
}

(Received in final form 17 July 1998)

\begin{abstract}
This series of two papers focuses on the determination and clarification of the characteristics of the forced response of bladed disks that exhibit a mistuning pattern that is either harmonic or partial. Harmonic mistuning refers to single wavelength variations in structural properties along the disk while partial mistuning is associated with blade characteristics that are random in a specific sector and tuned elsewhere. The results of this analysis demonstrate that many features of the response of these simple systems match not only qualitatively but also quantitatively their counterparts on randomly mistuned bladed disks. Relying on these similarities, simple and reliable approximations of the localization factor and of the mean response are easily derived that exemplify the usefulness of harmonic and partial mistuning patterns. Finally, it is demonstrated both theoretically and by comparison with simulation results that the maximum amplitude of response of a disk closely follows a Weibull-type distribution in all coupling situations, from very weak to very strong.
\end{abstract}

Keywords: Bladed disks, Blade vibration, Mistuning, Localization phenomenon, Maximum response

\section{INTRODUCTION}

The investigation of the effects of mistuning, i.e. of blade-to-blade variations in their geometry and structural properties that occur during the manufacturing process and/or as a consequence of inservice wear, has been ongoing for more than thirty years. These efforts have resulted in a large number of publications (see for example the works of Whitehead, 1966; Ewins, 1969, etc.) in which many qualitative and quantitative aspects of this problem have been clarified. The unpredictability of the exact properties, e.g. stiffnesses, masses, damping coefficients, of the blades of actual turbomachines has often led to the modeling of these structural characteristics as random variables with specified distributions and moments. The assessment of the damaging effects of mistuning then requires the determination of the probabilistic features of the forced response of these random disks, i.e. moments

\footnotetext{
* This paper was originally presented at ISROMAC-7.

${ }^{\dagger}$ Corresponding author. Tel.: (480) 965-1484. Fax: (480) 965-1384. E-mail: mignolet@enuxsa.eas.asu.edu.
} 
and distributions of the amplitude of the response of a typical blade and of the highest responding one. However, the inherent nonlinearity of the response of multi-degree-of-freedom systems with respect to their structural properties, i.e. stiffnesses, masses, and damping coefficients, renders the determination of the aforementioned moments and distributions a very challenging problem.

This situation has quite naturally led to the widespread use of Monte Carlo simulation (Basu and Griffin, 1986; Griffin and Hoosac, 1984) and perturbation methods (Sinha, 1986; Sinha and Chen, 1989; Wei and Pierre, 1988; Mignolet and Lin, 1993; 1996; Lin and Mignolet, 1997). Although these two techniques are quite different from a formulation point of view, they both provide "an approximate solution of the exact problem" with specific limitations. For example, perturbation methods have been found to yield different representations of the forced response in different ranges of values of the coupling strength between blades. On the contrary, the accuracy of Monte Carlo methods depends only slightly, if at all, on the specific values of the parameters of the problem but it does not provide any functional dependence, even approximate, of the features of the response on these parameters.

In this light, the present investigation can be considered a dual of earlier perturbation and Monte Carlo simulation studies since it focuses on obtaining "the exact solutions of approximate problems". Specifically, the exact forced response will be derived for two types of mistuned bladed disks, i.e. those whose structural properties vary harmonically around the disk (harmonic mistuning) and those which are tuned except for a few consecutive blades the properties of which vary randomly (partial mistuning). Interestingly, harmonic mistuning has already been considered in a few bladed disk analyses (see for example Valero and Bendiksen, 1986, and, for alternate mistuning, Kielb and Kaza, 1984; Kaza and Kielb, 1985), but only as an example of a mistuning pattern, not as a basis to thoroughly elucidate the effects of the localization phenomenon as is intended here. Specifically, both harmonic and partial mistuning and their corresponding forced responses will be related to the more standard case of a randomly mistuning bladed disk to provide new insights into the prediction of their forced response thereby complimenting perturbation and simulation analysis results.

\section{STRUCTURAL DYNAMIC MODELING}

For simplicity of the analysis, the one-degree-offreedom per blade model shown in Fig. 1 will be adopted in the sequel to represent the bladed disk. Previous investigations of this system (Sinha, 1986; Sinha and Chen, 1989; Wei and Pierre, 1988; Mignolet and Lin, 1993; 1996; Lin and Mignolet, 1997) have shown that this simple model does exhibit the high sensitivity of the forced response with respect to small fluctuations in the blade stiffnesses $\left(k_{j}\right)$ that is characteristic of mistuning. Following previous investigations (Sinha, 1986;

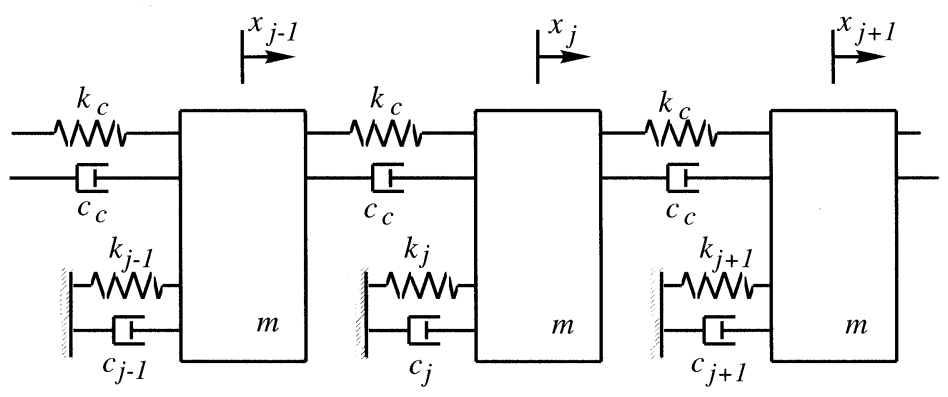

FIGURE 1 One degree-of-freedom per blade bladed disk model. 
Sinha and Chen, 1989), the 24 blades will be assumed to have the same masses $(m=0.0114 \mathrm{~kg})$, damping coefficients $\left(c_{j}=1.443 \mathrm{~N} \mathrm{~s} / \mathrm{m}\right.$, approximately $1 \%$ of the critical value), but have different stiffnesses. Specifically, the parameters $k_{j}$ are selected to be independent Gaussian random variables with mean $k_{t}=430,000 \mathrm{~N} / \mathrm{m}$ and standard deviation $\sigma_{k}=8,000 \mathrm{~N} / \mathrm{m}$. Further, the aerodynamic and structural coupling between blades is modeled by springs and dashpots of common values $k_{\mathrm{C}}$ and $c_{\mathrm{C}}$. In the present study, different values of $k_{\mathrm{C}}$ will be used but the parameter $c_{\mathrm{C}}$ is set to zero in all cases.

Then, the equation of motion of blade $j$, $j=1,2, \ldots, N$, can be written in the form

$$
\begin{aligned}
& m \ddot{X}_{j}+c \dot{X}_{j}+\left(k_{j}+2 k_{\mathrm{C}}\right) X_{j} \\
& \quad-k_{\mathrm{C}} X_{j-1}-k_{\mathrm{C}} X_{j+1}=F_{j}(t),
\end{aligned}
$$

where $F_{j}(t)$ represents the force acting on that blade. Of particular interest in the present investigation is an $r$ th engine order excitation of the form

$$
F_{j}(t)=\tilde{F}_{0} \mathrm{e}^{\mathrm{i}(\omega t+(j-1) \Delta \psi)} \quad j=1,2, \ldots, N,
$$

where $\omega$ and $\Delta \psi$ are the corresponding natural frequency and interblade phase angle of the tuned system, i.e.

$$
\omega=\omega_{r}=\sqrt{\frac{k_{t}+4 k_{\mathrm{C}} \sin ^{2}(\pi r / N)}{m}}
$$

and

$$
\Delta \psi=\frac{2 \pi}{N} r .
$$

The forced response of the $j$ th blade will be sought in the form

$$
X_{j}(t)=\tilde{X}_{j} \mathrm{e}^{\mathrm{i}(\omega t+(j-1) \Delta \psi)} \quad j=1,2, \ldots, N,
$$

where the symbols $\tilde{X}_{j}$ denote complex constants. Introducing this assumed solution in Eq. (1) yields the recurrence relation

$$
\begin{aligned}
& -k_{\mathrm{C}} \mathrm{e}^{\mathrm{i} \Delta \psi} \tilde{X}_{j+1}-k_{\mathrm{C}} \mathrm{e}^{-\mathrm{i} \Delta \psi} \tilde{X}_{j-1} \\
& \quad+\left(\delta k_{j}+2 k_{\mathrm{C}} \cos \Delta \psi+\mathrm{i} \omega c\right) \tilde{X}_{j}=\tilde{F}_{0} \\
& \quad j=1,2, \ldots, N,
\end{aligned}
$$

where $\delta k_{j}=k_{j}-k_{t}$. The solution of this equation must be obtained with the periodicity conditions

$$
\tilde{X}_{0}=\tilde{X}_{N} \quad \text { and } \quad \tilde{X}_{1}=\tilde{X}_{N+1} .
$$

Before studying some of the characteristics of the response of this mistuned system, it is appropriate to first obtain the basic features of its tuned counterpart, i.e. with $\delta k_{j}=0$. Of particular importance in the present investigation is its impulse response, i.e. the solution of

$$
\begin{aligned}
& -k_{\mathrm{C}} \mathrm{e}^{\mathrm{i} \Delta \psi} \tilde{h}_{j+1}-k_{\mathrm{C}} \mathrm{e}^{-\mathrm{i} \Delta \psi} \tilde{h}_{j-1} \\
& +\left(2 k_{\mathrm{C}} \cos \Delta \psi+\mathrm{i} \omega c\right) \tilde{h}_{j}=\delta_{j 1} \\
& \quad j=1,2, \ldots, N
\end{aligned}
$$

where $\delta_{j l}$ denotes the Kronecker symbol and with periodicity conditions similar to Eq. (7). The above nonhomogenous equation is equivalent to the homogenous recurrence

$$
\begin{aligned}
& -k_{\mathrm{C}} \mathrm{e}^{\mathrm{i} \Delta \psi} \tilde{h}_{j+1}-k_{\mathrm{C}} \mathrm{e}^{-\mathrm{i} \Delta \psi} \tilde{h}_{j-1} \\
& +\left(2 k_{\mathrm{C}} \cos \Delta \psi+\mathrm{i} \omega c\right) \tilde{h}_{j}=0 \\
& \quad j=2, \ldots, N
\end{aligned}
$$

with the boundary conditions

$$
\begin{aligned}
& -k_{\mathrm{C}} \mathrm{e}^{\mathrm{i} \Delta \psi} \tilde{h}_{2}-k_{\mathrm{C}} \mathrm{e}^{-\mathrm{i} \Delta \psi} \tilde{h}_{N} \\
& +\left(2 k_{\mathrm{C}} \cos \Delta \psi+\mathrm{i} \omega c\right) \tilde{h}_{1}=1
\end{aligned}
$$

and

$$
\tilde{h}_{N+1}=\tilde{h}_{1}
$$

Noting that the difference equation, Eq. (9), is characterized by constant coefficients, its solution can be sought as a linear combination of terms of 
the form $A \lambda^{j}$ for some parameters $A$ and $\lambda$. Proceeding in this manner, it can be shown that

$$
\tilde{h}_{j}=\frac{\mathrm{e}^{-\mathrm{i}(j-1) \Delta \psi} z_{0}}{k_{\mathrm{C}}\left(1-z_{0}^{2}\right)\left(1-z_{0}^{N}\right)}\left[z_{0}^{j-1}+z_{0}^{N-j+1}\right],
$$

where $z_{0}$ is any of the two complex solutions of the quadratic equation

$$
z_{0}^{2}-\frac{\left(2 k_{\mathrm{C}} \cos \Delta \psi+\mathrm{i} \omega c\right)}{k_{\mathrm{C}}} z_{0}+1=0
$$

that is

$$
z_{0}=\left(\cos \Delta \psi+\frac{\mathrm{i} \omega c}{2 k_{\mathrm{C}}}\right) \pm \sqrt{\left(\cos \Delta \psi+\frac{\mathrm{i} \omega c}{2 k_{\mathrm{C}}}\right)^{2}-1}
$$

At this point, note that the two roots of Eq. (13) are inverse of each other, i.e. $z_{0}$ and $1 / z_{0}$, and that the expression for the impulse response $\tilde{h}_{j}$ given in Eq. (12) is unaffected by the transformation $z_{0} \rightarrow 1 / z_{0}$. Thus, one can conventionally assume that the root $z_{0}$ has a magnitude $\left|z_{0}\right| \leq 1$ and this condition specifies the appropriate sign in Eq. (14).

In the absence of damping, $c \omega=0$, it is seen from Eq. (14) that $z_{0}=\mathrm{e}^{ \pm \mathrm{i} \Delta \psi}$ so that $\left|z_{0}\right|=1$. However, the presence of dissipation in the system yields a root $z_{0}$ such that $\left|z_{0}\right|<1$ and the magnitude $\left|z_{0}\right|$ decreases monotonically as the ratio $c \omega / k_{\mathrm{C}}$ increases. Then, when the number of blades, $N$, is large enough, it may be assumed that $\left|z_{0}\right|^{N} \ll 1$ and $\left|z_{0}\right|^{N-j+1} \ll 1, j$ not close to $N$, so that

$$
\tilde{h}_{j} \approx \frac{\mathrm{e}^{-\mathrm{i}(j-1) \Delta \psi} z_{0}^{j}}{k_{\mathrm{C}}\left(1-z_{0}^{2}\right)} .
$$

Combining this relation and the above observations, it is found as expected that damping creates an exponential decay of the propagation of the impulse response around the disk. Further, the exponential decay rate, $-\ln z_{0}$, increases monotonically with the parameter $c \omega / k_{\mathrm{C}}$.

\section{HARMONIC MISTUNING}

As already observed by Huang (1982), the structure of Eq. (6) is similar to the discretized version of the equation of motion of a single-degree-of-freedom with parametric excitation. Specifically, using the finite difference formula

$$
\frac{\mathrm{d}^{2} \tilde{X}(\theta)}{\mathrm{d} \theta^{2}} \approx \frac{\tilde{X}_{j+1}-2 \tilde{X}_{j}+\tilde{X}_{j-1}}{\Delta \theta^{2}}
$$

where $\theta=(2 \pi / N)(j-1)$ and $\Delta \theta=2 \pi / N$, in Eq. (6) yields the approximately equivalent differential equation

$$
\begin{aligned}
& -k_{\mathrm{C}} \Delta \theta^{2} \frac{\mathrm{d}^{2} \tilde{X}}{\mathrm{~d} \theta^{2}}-2 \mathrm{i} k_{\mathrm{C}} \Delta \theta \Delta \psi \frac{\mathrm{d} \tilde{X}}{\mathrm{~d} \theta} \\
& +\left[\delta k(\theta)+k_{\mathrm{C}}\left(\Delta \psi^{2}-4 \sin ^{2} \frac{\Delta \psi}{2}\right)+\mathrm{i} \omega c\right] \tilde{X} \\
& =\tilde{F}_{0} .
\end{aligned}
$$

The importance of Mathieu's equation in the analysis of parametrically excited systems suggests the expansion of the stiffnesses $\delta k_{j}$ in Fourier series, i.e.

$$
\begin{aligned}
\delta k_{j} & =\sum_{s=0}^{N-1} \beta_{s} \mathrm{e}^{-\mathrm{i}(2 \pi / N) s(j-1)} \text { and } \\
\beta_{s} & =\frac{1}{N} \sum_{j=1}^{N} \delta k_{j} \mathrm{e}^{\mathrm{i}(2 \pi / N) s(j-1)} .
\end{aligned}
$$

Note from the above equations that the real character of the stiffnesses $\delta k_{j}$ implies that $\beta_{s}^{*}=$ $\beta_{N-s}$ where $*$ denotes the operation of complex conjugation. Further, if the random variables $\delta k_{j}$ are independent of each other, then so are the coefficients $\beta_{s}$ and

$$
E\left[\left|\beta_{s}\right|^{2}\right]=\frac{1}{N} E\left[\delta k_{j}^{2}\right]=\frac{\sigma_{k}^{2}}{N}
$$


Combining Eqs. (6) and (18), yields the governing equation for the response of the system as

$$
\begin{aligned}
& -k_{\mathrm{C}} \mathrm{e}^{\mathrm{i} \Delta \psi} \tilde{X}_{j+1}-k_{\mathrm{C}} \mathrm{e}^{-\mathrm{i} \Delta \psi} \tilde{X}_{j-1} \\
& +\left(2 k_{\mathrm{C}} \cos \Delta \psi+\mathrm{i} \omega c+\sum_{s=0}^{N-1} \beta_{s} \mathrm{e}^{-\mathrm{i}(2 \pi / N) s(j-1)}\right) \tilde{X}_{j} \\
& \quad=\tilde{F}_{0} .
\end{aligned}
$$

To gain insight into the effects of the small Fourier coefficients $\beta_{s}$ on the forced response, a straightforward perturbation analysis has been carried out assuming that $\beta_{s} \ll k_{\mathrm{C}}, c \omega$. Accordingly, the forced response of the $j$ th blade can be written in the form

$$
\begin{aligned}
\tilde{X}_{j} & =\frac{\tilde{F}_{0}}{\mathrm{i} c \omega}+\frac{\tilde{F}_{0}}{\mathrm{i} c \omega} \\
& \times \sum_{s=0}^{N-1} \frac{\beta_{s} \mathrm{e}^{-\mathrm{i}(2 \pi / N) s(j-1)}}{\mathrm{i} c \omega+2 k_{\mathrm{C}}(\cos (2 \pi r / N)-\cos (2 \pi(r-s) / N))}
\end{aligned}
$$

to first order in the small coefficients $\beta_{s}$. Note from the above relation that the effect of the mistuning harmonic of order $s, \beta_{s}$, is modulated by the inverse of term ic $\omega+2 k_{C}(\cos (2 \pi r / N)-$ $\cos (2 \pi(r-s) / N))$. When the coupling stiffness is much larger than the damping term $c \omega$, it is seen that the summation is dominated by the terms corresponding to $s=0$ and $s=2 r$ which yield contributions of the order of $1 / i c \omega$.

This result can be explained physically as follows. The engine order excitation, Eqs. (2)-(4), is in fact a resonant traveling wave which would create on a tuned bladed disk a response of the same form, i.e. given by Eq. (5) with $\tilde{X}_{j}=$ constant. Then, a perturbation of the mean stiffness of the blades, i.e., $\beta_{0}$, alters the natural frequencies of the system so that the forcing excitation becomes off-resonant. This relative shift of the natural and excitation frequencies is thus accompanied by a large decrease in the response of the blades, as observed from Eq. (21), since the modal damping ratios are small.
The preeminence of the terms $s=2 r$ in Eq. (21) is not associated, unlike the $s=0$ contribution, with the excitation wave but rather with its reflections which are naturally created by the dissimilarities in the blade properties. These reflections (backward wave) are characterized by the same frequency as the excitation (forward wave) and a magnitude that is directly related to the dissimilarities in the blade properties. Thus, in the presence of random mistuning $\delta k_{j}$, Eq. (18), the combination of reflections created at each blade-to-blade interface exhibits all negative interblade phase angles when expanded in Fourier series. Of primary effect on the forced response is the component of this backward wave associated with the interblade phase angle $\Delta \psi^{\prime}=-(2 \pi / N) r$ which induces, as its forward component, $\Delta \psi=(2 \pi / N) r$, resonance in the system. This excitation is the result of the fluctuations in the stiffnesses of the blades and their response to the forward wave, i.e. it is associated with the product $\delta k_{j} X_{j}(t)$. Equivalently, the backward wave will be generated when

$$
\begin{aligned}
\delta k_{j} X_{j}(t) & =\delta k_{j}\left[\frac{\tilde{F}_{0}}{\mathrm{i} \omega c} \mathrm{e}^{\mathrm{i}(\omega t+(j-1) \Delta \psi)}\right] \\
& =\tilde{Y}_{0} \mathrm{e}^{\mathrm{i}(\omega t-(j-1) \Delta \psi)}
\end{aligned}
$$

for some complex number $\tilde{Y}_{0}$. The above relation yields the critical mistuning pattern as

$$
\delta k_{j}=\beta_{2 r} \mathrm{e}^{-\mathrm{i} 2(j-1) \Delta \psi}=\beta_{2 r} \mathrm{e}^{-\mathrm{i}(j-1)(2 \pi / N)(2 r)}
$$

which is exactly of the form of Eq. (18) with $s=2 r$ thereby confirming the importance of the $\beta_{2 r}$ term in Eq. (21).

The above findings have been obtained under the assumption $k_{\mathrm{C}} \gg c \omega$ which is required for the first order perturbation result, Eq. (21), to hold. To assess the validity of these observations for $k_{\mathrm{C}} \in[0, \infty)$, the exact forced response of the bladed disk was computed in the presence of a single harmonic of mistuning

$$
\begin{aligned}
\delta k_{j} & =\beta_{s} \mathrm{e}^{-\mathrm{i}(2 \pi / N) s(j-1)}+\beta_{N-s} \mathrm{e}^{\mathrm{i}(2 \pi / N) s(j-1)} \\
& =\left|\beta_{s}\right| \cos \left[\frac{2 \pi}{N} s(j-1)+\phi_{s}\right]
\end{aligned}
$$


with, $\left|\beta_{s}\right|=(8,000 / \sqrt{24}) \mathrm{N} / \mathrm{m}, \tilde{F}_{0}=1 \mathrm{~N}$, and $r=3$ for different values of $k_{\mathrm{C}}$. Interestingly, it was found that neither the mean amplitude observed over the bladed disk nor the corresponding root mean square value were functions of the phase $\phi_{s}$ of the stiffness mistuning distribution. However, their variations with the harmonic number $s$ for three typical values of the coupling stiffness, $k_{\mathrm{C}}$, are shown in Figs. 2 and 3. Clearly, for values of this parameter much larger than the damping term $(c \omega \approx 8,000 \mathrm{~N} / \mathrm{m})$ and the mistuning strength $\left(\left|\beta_{s}\right|=1,633 \mathrm{~N} / \mathrm{m}\right)$, i.e. for $k_{\mathrm{C}}=45,430 \mathrm{~N} / \mathrm{m}$ in Figs. 2 and 3, both the mean and root mean square values of the amplitude exhibit sharp peaks at the expected values of the harmonic number, that is at $s=0, s=2 r$, and $s=N-2 r$.

As the blade-to-blade coupling is reduced, keeping the damping level and the mistuning strength constant, it is seen that the peaks have decreased substantially in magnitude and have broadened to include not only the values $s=2 r$ and $s=N-2 r$ but also the neighborhooding harmonic numbers, see Figs. 2 and 3 for $k_{\mathrm{C}}=$ $8,000 \mathrm{~N} / \mathrm{m}$. This result is still consistent with the first order perturbation results given by Eq. (21). Specifically, the range of values of the harmonic number $s$ for which the effects of the coupling in the magnitude of the term $i \omega c+2 k_{\mathrm{C}}[\cos (2 \pi r / N)$ $-\cos (2 \pi(r-s) / N)]$ is small expands from $s=2 r$ only for $k_{\mathrm{C}} \gg c \omega$ to $s \in[2 r-1,2 r+1]$, then, $s \in[2 r-2,2 r+2]$, etc., as $k_{\mathrm{C}}$ decreases.

Reducing further the coupling stiffness to $1,000 \mathrm{~N} / \mathrm{m}$ leads to a rather different situation, as seen in Figs. 2 and 3. Specifically, it is seen that the mean value of the amplitude of response is almost independent of the harmonic order $s>0$ except for $s=N / 2 \quad(=12)$ value at which a sharp peak is encountered. A similar, but less dramatic, situation can be observed in the plot of the root mean square of the amplitude. At the contrary of the previous observations, this finding cannot be explained by investigating Eq. (21) since this equation

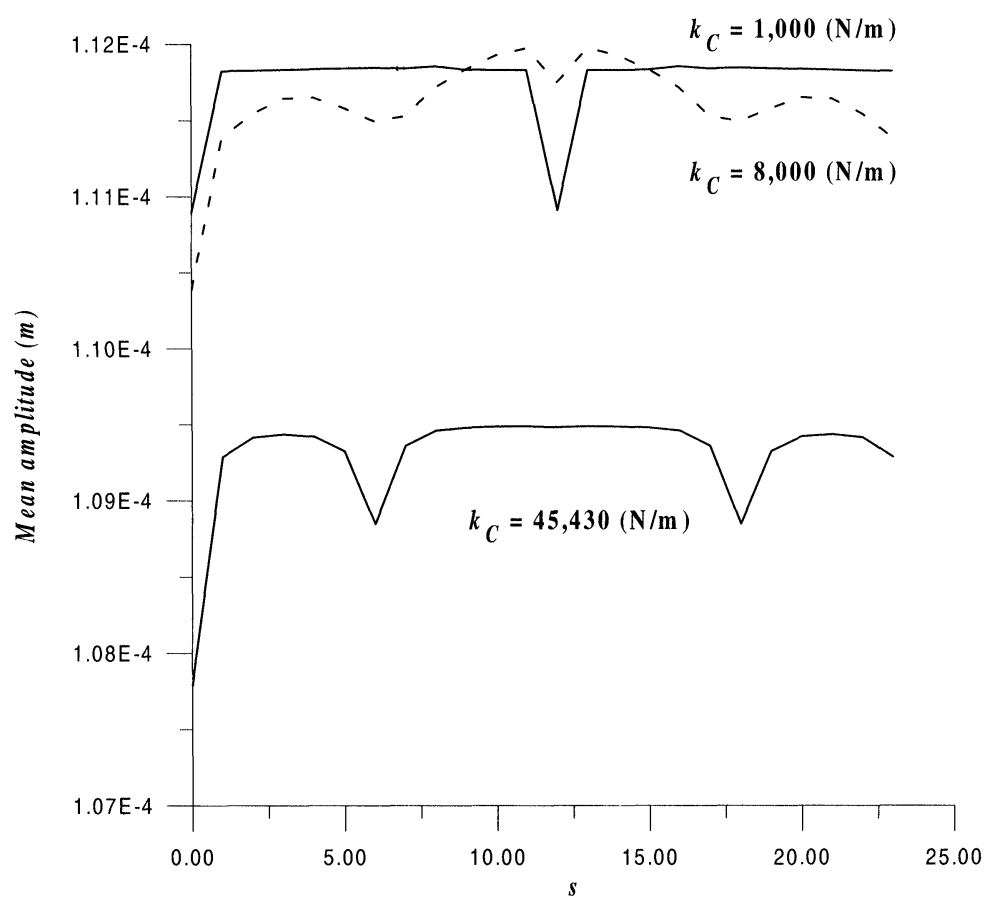

FIGURE 2 Mean amplitude of response as a function of harmonic number for different coupling stiffnesses, $\left|\beta_{s}\right|=$ $1,633 \mathrm{~N} / \mathrm{m}, c=1.443 \mathrm{Ns} / \mathrm{m}$. 


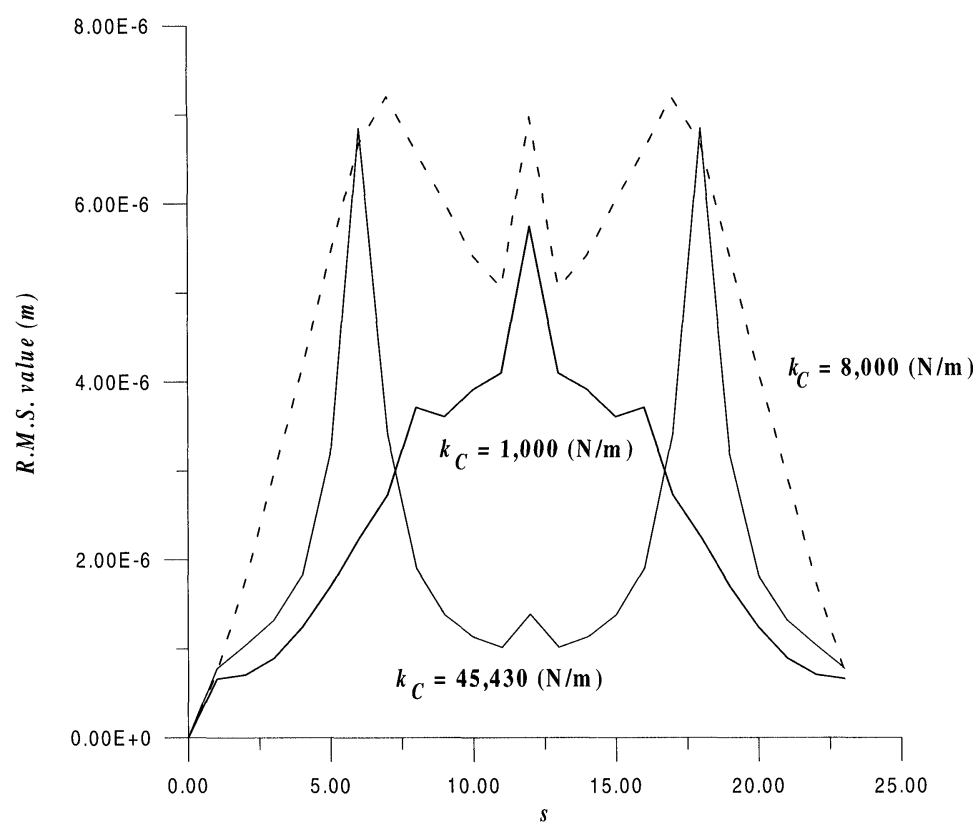

FIGURE 3 Root mean square amplitude of response as a function of harmonic number for different coupling stiffnesses, $\left|\beta_{s}\right|=1,633 \mathrm{~N} / \mathrm{m}, c=1.443 \mathrm{Ns} / \mathrm{m}$.

would provide a similar effect of all harmonic numbers $s$. This situation is in fact not surprising since the validity of Eq. (21) is limited to the cases where $k_{\mathrm{C}} \gg c \omega, \beta_{s}$ which is clearly not true here since $\beta_{s}(=1,633 \mathrm{~N} / \mathrm{m})$ and $c \omega(=8,868.38 \mathrm{~N} / \mathrm{m})$ are both larger than $k_{\mathrm{C}}(=1,000 \mathrm{~N} / \mathrm{m})$. Nevertheless, the preeminence of the harmonic orders $s \approx N / 2$ can be explained on physical grounds; it is symptomatic of a strong localization of vibration in the system (Wei and Pierre, 1988; Castanier and Pierre, 1993). That is, the reflected waves shed at each blade-toblade interface with dissimilar properties rapidly decay as they propagate along the disk. Then, the effects of fluctuations in blade characteristics with long wavelengths which consist of slowly varying changes in the amplitude of vibration of the blades will be damped before they produce a large variability in the response. Thus, it is the short wavelength changes in stiffness, i.e. those associated with harmonic numbers $s$ close to $N / 2$, that will have the largest effect on the dynamic behavior of the system. Additional physical understanding of this situation can be obtained by relying on a recent analysis (Castanier and Pierre, 1993) of the localization phenomenon in damped nearly periodic structures. Although the present investigation focuses on cyclic assemblies, the strong localization results derived therein are applicable in the present context since localization prevents the flow of "information" around the disk so that the cyclic character of the system only has a minor influence on its response.

The study of Castanier and Pierre (1993), has revealed the critical role of the two parameters $\sigma / R$ and $\delta / R$ where

$$
\begin{aligned}
\sigma & =\frac{\beta_{s}}{k_{t}}\left(\frac{1}{2}+\frac{1}{2} \delta_{s 0}+\frac{1}{2} \delta_{s \frac{N}{2}}\right), \\
R & =\frac{k_{\mathrm{C}}}{k_{t}} \text { and } \delta=\frac{c \omega}{k_{t}}
\end{aligned}
$$

so that

$$
\frac{\sigma}{R}=\frac{\beta_{s}}{k_{\mathrm{C}}}\left(\frac{1}{2}+\frac{1}{2} \delta_{s 0}+\frac{1}{2} \delta_{s \frac{N}{2}}\right) \quad \text { and } \quad \frac{\delta}{R}=\frac{c \omega}{k_{\mathrm{C}}} .
$$


Specifically, it was demonstrated in that study that strong localization occurs when either $\delta / R \gg 1$ or $\sigma / R \gg 1$. With the values $\beta_{s}=1,633 \mathrm{~N} / \mathrm{m}$ and $c \omega=$ $8,868.38 \mathrm{~N} / \mathrm{m}$ used in obtaining the results shown in Figs. 2 and 3, it is found that $\sigma / R=1.63$ and $\delta / R=8.87$ for $k_{\mathrm{C}}=1,000 \mathrm{~N} / \mathrm{m}$ and the strong localization character of the corresponding response is confirmed. The other cases investigated in Figs. 2 and 3 , i.e. $k_{\mathrm{C}}=8,000 \mathrm{~N} / \mathrm{m}$ and $45,430 \mathrm{~N} / \mathrm{m}$, correspond to the values $(\sigma / R, \delta / R)=(0.204,1.114)$ and $(0.036,0.201)$, respectively, which can both be classified as weak to moderate localization situations. Thus, to obtain a complete picture of the effects of localization in harmonically mistuned bladed disks, it is necessary to also investigate the cases in which one of the ratios $\delta / R$ or $\sigma / R$ is much larger than unity but the other one is not.

To this end, the coupling stiffness was kept as $k_{\mathrm{C}}=1,000 \mathrm{~N} / \mathrm{m}$ and the mistuning strength $\beta_{s}$ was reduced to a tenth of its previous value, i.e. $\beta_{s}=163.3 \mathrm{~N} / \mathrm{m}$, so that $\sigma / R=0.163<1$. Shown in Figs. 4 and 5 are the mean and root mean square amplitudes of the response for the values $c \omega=$ $8,868.38 \mathrm{~N} / \mathrm{m}, \delta / R=8.868$ (curve labeled (2)), and $c \omega=886.84 \mathrm{~N} / \mathrm{m}, \delta / R=0.887$ (curve labeled (3)). Also displayed in these figures and labeled as (1) are the curves corresponding to $k_{\mathrm{C}}=1,000 \mathrm{~N} / \mathrm{m}$ shown in Figs. 2 and 3. Comparing the curves (1) and (2), it is seen that the decrease in mistuning strength in this system strongly localized by damping has not changed the physical characteristics of the forced response, i.e. the preeminence of the harmonics, $s \approx N / 2$, it has only changed the magnitudes of their effects.

Reducing the damping term $c \omega$ to $886.84 \mathrm{~N} / \mathrm{m}$ $(0.1 \%$ of critical) yields the curves labeled (3) which are very similar those seen in Figs. 2 and 3 in connection with $k_{\mathrm{C}}=8,000 \mathrm{~N} / \mathrm{m}$. This result is
(1) $+(2)$

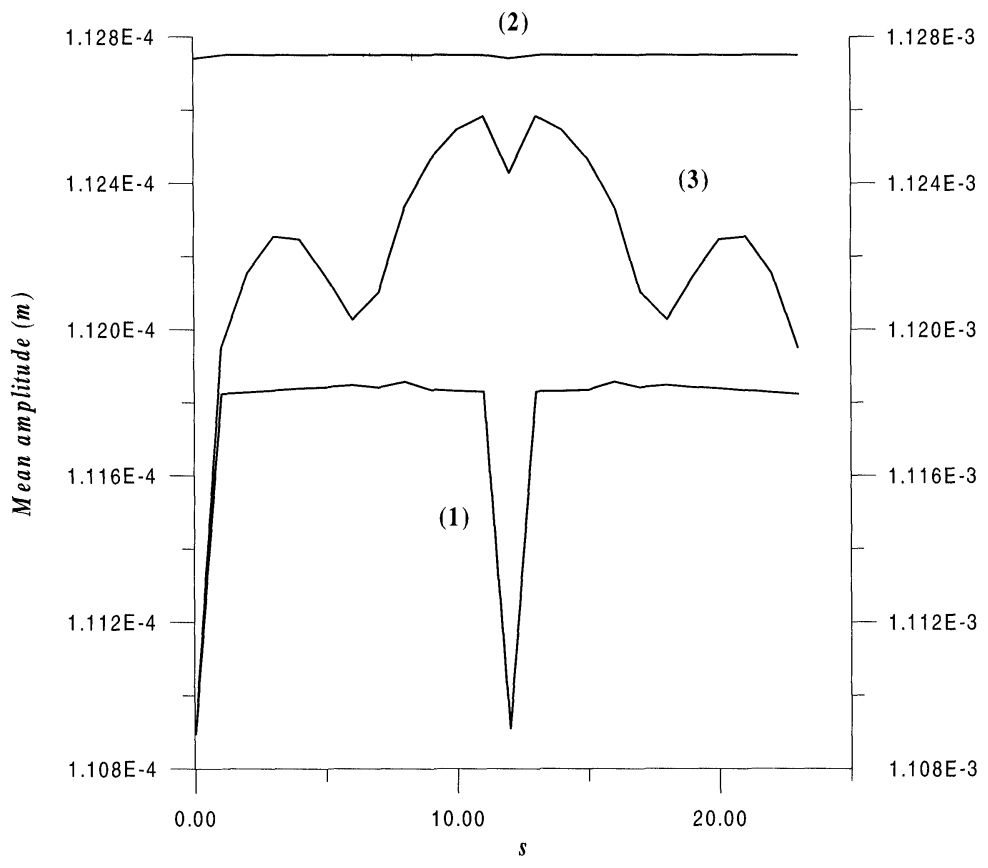

FIGURE 4 Mean amplitude of response as a function of harmonic number. Case (1) (left axis): $k_{\mathrm{C}}=1,000 \mathrm{~N} / \mathrm{m},\left|\beta_{s}\right|=1,633 \mathrm{~N} /$ $\mathrm{m}, c=1.443 \mathrm{Ns} / \mathrm{m}$; Case (2) (left axis): $k_{\mathrm{C}}=1,000 \mathrm{~N} / \mathrm{m},\left|\beta_{s}\right|=163.3 \mathrm{~N} / \mathrm{m}, c=1.443 \mathrm{Ns} / \mathrm{m}$; Case (3) (right axis): $k_{\mathrm{C}}=1,000 \mathrm{~N} / \mathrm{m}$, $\beta_{s}=163.3 \mathrm{~N} / \mathrm{m}, c=0.1443 \mathrm{Ns} / \mathrm{m}$. 


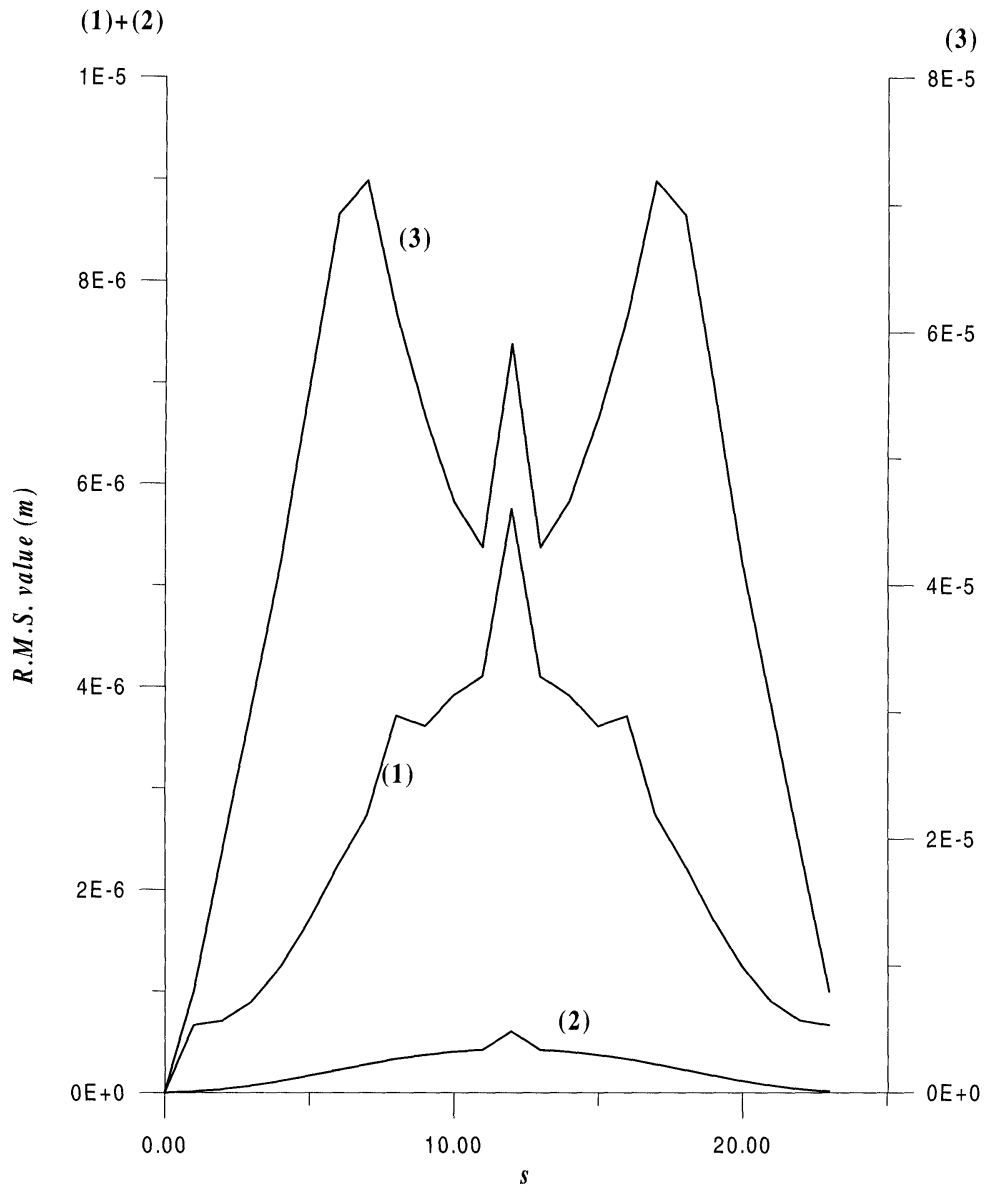

FIGURE 5 Root mean square amplitude of response as a function of harmonic number. Case (1) (left axis): $k_{\mathrm{C}}=1,000 \mathrm{~N} / \mathrm{m}$, $\left|\beta_{s}\right|=1,633 \mathrm{~N} / \mathrm{m}, c=1.443 \mathrm{Ns} / \mathrm{m}$; Case (2) (left axis): $k_{\mathrm{C}}=1,000 \mathrm{~N} / \mathrm{m},\left|\beta_{s}\right|=163.3 \mathrm{~N} / \mathrm{m}, c=1.443 \mathrm{Ns} / \mathrm{m}$; Case (3) (right axis): $k_{\mathrm{C}}=$ $1,000 \mathrm{~N} / \mathrm{m} ;\left|\beta_{s}\right|=163.3 \mathrm{~N} / \mathrm{m}, c=0.1443 \mathrm{Ns} / \mathrm{m}$.

expected since the corresponding values of $\sigma / R$ and $\delta / R$ are 0.163 and 0.887 , respectively, which are representative of a mildly localized disk.

To investigate the dominance of the effects of the localization associated with mistuning, the coupling stiffness was again set as $k_{\mathrm{C}}=1,000 \mathrm{~N} / \mathrm{m}$ but the mistuning strength was selected to be $\beta_{s}=8,000$ $\mathrm{N} / \mathrm{m}$, so that $\sigma / R=8.00$. Shown in Figs. 6 and 7 are the mean and root mean square amplitudes of the response as functions of the mistuning harmonic number for the values $c \omega=8,868.38 \mathrm{~N} / \mathrm{m}$ (curve labeled (4)), and $c \omega=886.84 \mathrm{~N} / \mathrm{m}$ (curve labeled (5)). In the first case, for which $\delta / R=8.868$ so that $\delta / R \approx \sigma / R$, it is found that the overall features of the mean and root mean square values of the amplitude of response are very similar to those shown in Figs. 2 and $3, k_{\mathrm{C}}=1,000 \mathrm{~N} / \mathrm{m}$ case. However, there are some important differences between these sets of curves. Specifically, although the increase in the strength of mistuning has increased the localization in the system, it is seen that the response exhibits an increased sensitivity with respect to the harmonics $s=4,6$, and 8 of the mistuning pattern. These components which have period of 3, 4, and 6 blades correspond to longer range interactions than their $s=N / 2=12$ counterpart which was found dominant when $\delta / R>\sigma / R$ and $\delta / R \gg 1$. In fact, increasing further the strength of mistuning leads to even 
(5)

(4)

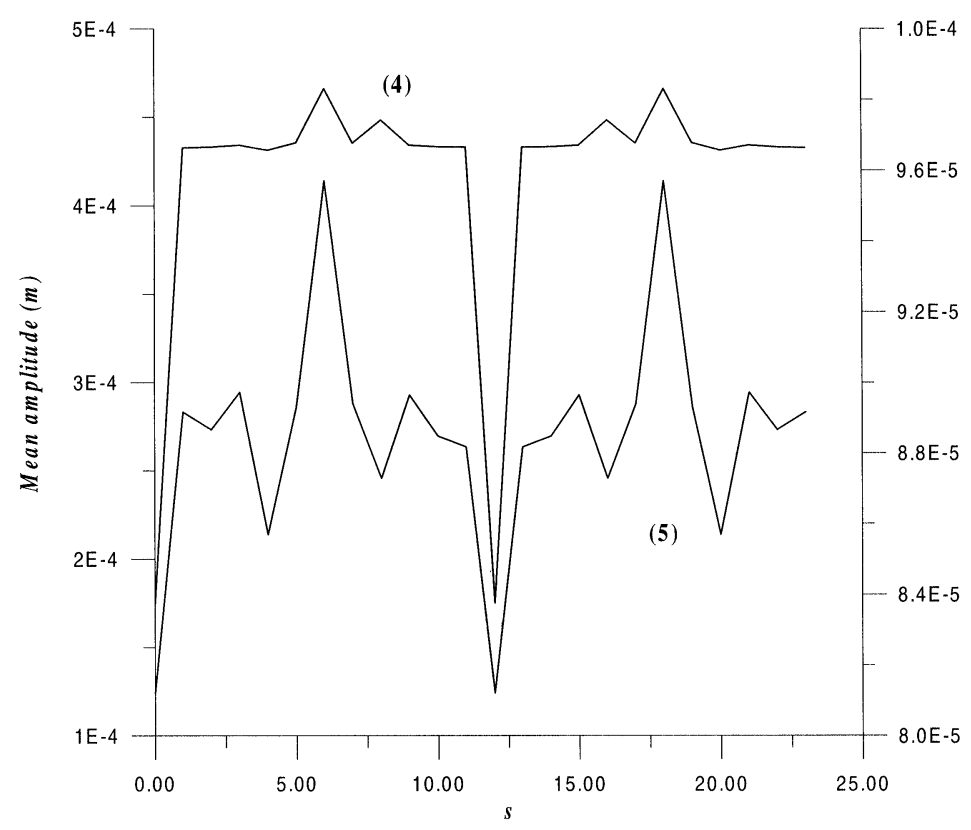

FIGURE 6 Mean amplitude of response as a function of harmonic number. Case (4) (right axis): $k_{\mathrm{C}}=1,000 \mathrm{~N} / \mathrm{m},\left|\beta_{s}\right|=8,000 \mathrm{~N} / \mathrm{m}$ $c=1.443 \mathrm{Ns} / \mathrm{m}$; Case (5) (left axis): $k_{\mathrm{C}}=1,000 \mathrm{~N} / \mathrm{m},\left|\beta_{s}\right|=8,000 \mathrm{~N} / \mathrm{m}, c=0.1443 \mathrm{Ns} / \mathrm{m}$

higher peaks at $s=4,6$, and $8(N / 6, N / 4$, and $N / 3)$ and a reduced one at $s=12(\mathrm{~N} / 2)$.

From the above sets of observations, it is concluded that the strong localization due to damping leads to a response which is most sensitive to the shortest wavelength fluctuations in the structural properties. Strong localization that is mostly generated by mistuning leads to a special sensitivity of the response with respect to the discrete set of wavelengths that are integer divisors of the number of blades in the disk. Moreover, the sensitivity of the response is largest for the smallest divisors.

This observed evolution, with changing coupling stiffness, of the characteristics (harmonics) of the mistuning pattern that are most dominant in the forced response of the disk closely parallels the findings of a recent investigation by Lin and Mignolet (1997). Specifically, it was demonstrated in that study that the largest modal contributions in the response of the system are associated with the modes of the tuned system whose natural frequencies are closest to the excitation frequency when the coupling stiffness is large. Further, as this param- eter decreases, the number of modes that have a strong presence in the distribution of the blade amplitudes increases. Finally, similar observations were also found to hold in the weakly coupling limit $k_{\mathrm{C}} \ll c \omega$ provided that the modes of the uncoupled system are used.

In this earlier paper, these findings were then used to formulate a novel adaptive perturbation strategy in which the dominant modal contributions were accurately computed while the remaining ones were approximated. The excellent results obtained with this approach and the similarities between this study and the present investigation suggest reformulating the governing equation for the response of the system, Eq. (6), in the form

$$
\begin{aligned}
& -k_{\mathrm{C}} \mathrm{e}^{\mathrm{i} \Delta \psi} \tilde{X}_{j+1}-k_{\mathrm{C}} \mathrm{e}^{-\mathrm{i} \Delta \psi} \tilde{X}_{j-1} \\
& +\left(\sum_{s \in S_{1}} \beta_{s} \mathrm{e}^{-\mathrm{i}(2 \pi / N) s(j-1)}+2 k_{\mathrm{C}} \cos \Delta \psi+\mathrm{i} \omega c\right) \tilde{X}_{j} \\
& \quad=\tilde{F}_{0}-\left(\sum_{s \in S_{2}} \beta_{s} \mathrm{e}^{-\mathrm{i}(2 \pi / N) s(j-1)}\right) \tilde{X}_{j},
\end{aligned}
$$




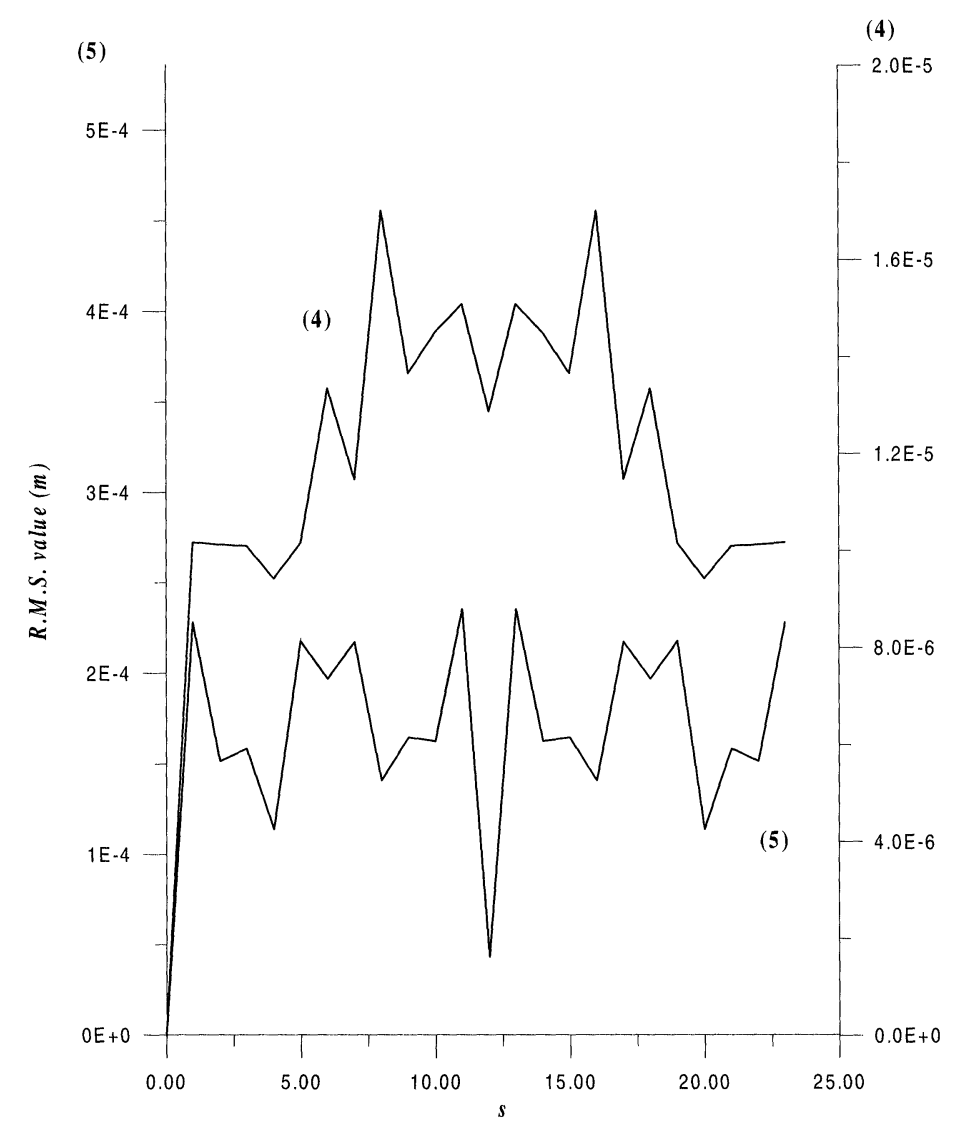

FIGURE 7 Root mean square amplitude of response as a function of harmonic number. Case (4) (right axis): $k_{\mathrm{C}}=1,000 \mathrm{~N} / \mathrm{m}$, $\left|\beta_{s}\right|=8,000 \mathrm{~N} / \mathrm{m}, c=1.443 \mathrm{Ns} / \mathrm{m}$; Case (5) (left axis): $k_{\mathrm{C}}=1,000 \mathrm{~N} / \mathrm{m},\left|\beta_{s}\right|=8,000 \mathrm{~N} / \mathrm{m}, c=0.1443 \mathrm{Ns} / \mathrm{m}$.

where $S_{1} \equiv[0, q] \cup[N-q, N-1] \cup[2 r-p, 2 r+p] \cup$ $[N-2 r-p, N-2 r+p]$ and $S_{2} \equiv[0, N-1]-S_{1}$ for some integers $p$ and $q$. Then, for mild to strong coupling, it is proposed to treat the effect of the mistuning harmonics $s \in S_{2}$ by perturbation while accounting exactly for the dependence of the forced response on the terms $\beta_{s}, s \in S_{1}$. That is, the solution of Eq. (27) will be sought in the form

$$
\tilde{X}_{j}=\tilde{X}_{j}^{(0)}+\tilde{X}_{j}^{(1)}+\cdots,
$$

where

$$
\begin{aligned}
& -k_{\mathrm{C}} \mathrm{e}^{\mathrm{i} \Delta \psi} \tilde{X}_{j+1}^{(0)}-k_{\mathrm{C}} \mathrm{e}^{-\mathrm{i} \Delta \psi} \tilde{X}_{j-1}^{(0)} \\
& +\left(\sum_{s \in S_{1}} \beta_{s} \mathrm{e}^{-\mathrm{i}(2 \pi / N) s(j-1)}+2 k_{\mathrm{C}} \cos \Delta \psi+\mathrm{i} \omega c\right) \tilde{X}_{j}^{(0)} \\
& =\tilde{F}_{0}
\end{aligned}
$$

and

$$
\begin{aligned}
& -k_{\mathrm{C}} \mathrm{e}^{\mathrm{i} \Delta \psi} \tilde{X}_{j+1}^{(l)}-k_{\mathrm{C}} \mathrm{e}^{-\mathrm{i} \Delta \psi} \tilde{X}_{j-1}^{(l)} \\
& +\left(\sum_{s \in S_{1}} \beta_{s} \mathrm{e}^{-\mathrm{i}(2 \pi / N) s(j-1)}+2 k_{\mathrm{C}} \cos \Delta \psi+\mathrm{i} \omega c\right) \tilde{X}_{j}^{(l)} \\
& \quad=-\left(\sum_{s \in S_{2}} \beta_{s} \mathrm{e}^{-\mathrm{i}(2 \pi / N) s(j-1)}\right) \tilde{X}_{j}^{(l-1)}
\end{aligned}
$$

for $l>1$. Further, for sufficiently large values of $k_{\mathrm{C}}$, it is sufficient to consider only the harmonics $s=0,2 r$, and $N-2 r$ in the set $S_{1}$ yielding the Mathieu's difference equation

$$
\begin{gathered}
\left(\beta_{s} \mathrm{e}^{-\mathrm{i}(2 \pi / N) s(j-1)}+\beta_{N-s} \mathrm{e}^{\mathrm{i}(2 \pi / N) s(j-1)}+\beta_{0}\right. \\
\left.+2 k_{\mathrm{C}} \cos \Delta \psi+\mathrm{i} \omega c\right) \tilde{X}_{j}^{(0)}-k_{\mathrm{C}} \mathrm{e}^{\mathrm{i} \Delta \psi} \tilde{X}_{j+1}^{(0)} \\
-k_{\mathrm{C}} \mathrm{e}^{-\mathrm{i} \Delta \psi} \tilde{X}_{j-1}^{(0)}=\tilde{F}_{0} \quad j=1,2, \ldots, N
\end{gathered}
$$


with $s=2 r$. When the ratio $\nu=N / s$ is an integer, it is seen that the above equation describes the response of a bladed disk which is composed of $s$ perfectly identical sets of $\nu$ blades. In this case, the periodicity of this system implies that $\tilde{X}_{j+v}^{(0)}=\tilde{X}_{j}^{(0)}$ and the response of the bladed disk requires only the determination of $\tilde{X}_{j}^{(0)}, j=1,2, \ldots, \nu$. This reduced set of unknowns produces a computational savings that can be dramatic especially for small values of $\nu$.

When $\nu=N / s$ is not an integer, the forced response of the disk is periodic only with period $N$ but still can be expressed as

$$
\tilde{X}_{j}^{(0)}=\sum_{n=0}^{m-1} \tilde{Y}_{n}^{(0)} \mathrm{e}^{\mathrm{i}(2 \pi / N) s n(j-1)}
$$

with

$$
m=\frac{1}{s} \operatorname{lcm}(N, s),
$$

where $\operatorname{lcm}(N, s)$ is the least common multiple of $N$ and $s$. Introducing Eq. (32) in Eq. (31) yields a system of equations for the parameters $\tilde{Y}_{n}^{(0)}$ in the form

$$
\begin{aligned}
& \left(-k_{\mathrm{C}} \mathrm{e}^{\mathrm{i} \Delta \psi} \varepsilon^{n}-k_{\mathrm{C}} \mathrm{e}^{-\mathrm{i} \Delta \psi} \varepsilon^{-n}+\beta_{0}\right. \\
& \left.\quad+2 k_{\mathrm{C}} \cos \Delta \psi+\mathrm{i} \omega c\right) \tilde{Y}_{n}^{(0)}+\beta_{N-s} \tilde{Y}_{n+1}^{(0)}+\beta_{s} \tilde{Y}_{n-1}^{(0)} \\
& \quad=\tilde{F}_{0} \delta_{n 0} \quad n=0,1,2, \ldots, m,
\end{aligned}
$$

where $\varepsilon=\mathrm{e}^{\mathrm{i}(2 \pi / N) s}$. Note the extreme similarity between this equation and Eq. (6) with the following important differences:

(i) The variables $\tilde{Y}_{n}^{(0)}$ denote the Fourier coefficients of the response not the response itself as in Eq. (6).

(ii) The roles of the coupling and mistuning are reversed; the response parameters $\tilde{Y}_{n}^{(0)}$ and $\tilde{Y}_{n \pm 1}^{(0)}$ are related to each other through the mistuning terms $\beta_{s}$ and $\beta_{N-s}$, not $k_{\mathrm{C}}$ as in Eq. (6), while the variations of the parameters of the above difference equation are associated with the coupling stiffness $k_{\mathrm{C}}$, not with $\beta_{s}$ and $\beta_{N-s}$ as in Eq. (6).
The above findings demonstrate that there exists a deep rooted duality between mistuning and coupling: the connection between the response of neighborhooding blades is provided by the structural coupling while mistuning links their Fourier components, or equivalently the modes of the tuned system. This observation provides new insight into the successes achieved with the adaptive perturbation technique (Lin and Mignolet, 1997). That is, when the coupling is strong, the response is localized to a few modes of the tuned system and a very reliable approximation of the amplitudes can be obtained by including a few modal components. On the contrary, when the coupling is weak, the response is localized to a few blades and the modes of the decoupled system clearly represent the dynamics of the system.

\section{SUMMARY}

In this first paper, the exact characteristics of harmonically mistuned bladed disks have been investigated to provide phenomenological insights into the effects of full random mistuning on the response of these systems. The contributions of this part of the investigation can be summarized as follows.

(1) The features of the mistuning pattern that most seriously affect the resonant forced response have been highlighted and their dependency on the level of blade-to-blade coupling has been elucidated. In particular, it was demonstrated that strongly coupled bladed disks are especially sensitive to harmonic fluctuations in the structural properties of the blades that correspond to a wave propagating in the tuned system at the same frequency as the excitation but in the reverse direction (backward wave). As the level of coupling is reduced, the preeminence of this Fourier component is reduced and the short wavelength harmonics of the mistuning pattern produce increasing fluctuations of the response of the disk. 
(2) The presence of strong localization effects created by a small level of structural damping ( $1 \%$ of critical) has been demonstrated for the first time and the differences between the forced responses of bladed disks strongly localized primarily by damping or by mistuning have been presented. Specifically, it was concluded that the strong localization due to damping leads to a response which is most sensitive to the shortest wavelength fluctuations in the structural properties. Strong localization that is mostly generated by mistuning leads to a special sensitivity of the response with respect to the discrete set of wavelengths that are integer divisors of the number of blades in the disk. Moreover, the sensitivity of the response is largest for the smallest divisors.

(3) The level of blade-to-blade coupling and the strength of mistuning were shown to play dual roles of each other. Specifically, the connection between the response of neighborhooding blades is provided by the structural coupling while mistuning links their Fourier components, or equivalently the modes of the tuned system. This observation indicates that when the coupling is strong, the response is localized to a few modes of the tuned system and a very reliable approximation of the amplitudes can be obtained by including a few modal components. On the contrary, when the coupling is weak, the response is localized to a few blades and the modes of the decoupled system clearly represent the dynamics of the system.

\section{Acknowledgment}

The financial support of this work by the Naval Surface Warfare Center, Caderock Division, Department of the Navy, Bethesda, MD 20084, is gratefully acknowledged. Further, the first author
(M.P.M.) wishes to express his deep gratitude to Prof. J.C.P. Mignolet for his continued support of this and other research efforts.

\section{References}

Basu, P. and Griffin, J.H., 1986. The effects of limiting aerodynamic and structural coupling in models of mistuned bladed disk vibration, Journal of Vibration, Acoustics, Stress, and Reliability in Design, 108, 132-139.

Castanier, M.P. and Pierre, C., 1993. Individual and interactive mechanisms for localization and dissipation in a monocoupled nearly-periodic structure, Journal of Sound and Vibration, 168, 479-505.

Ewins, D.J., 1969. The effects of detuning upon the forced vibrations of bladed disks, Journal of Sound and Vibration, 9 , 65-79.

Griffin, J.H. and Hoosac, T.M., 1984. Model development and statistical investigation of turbine blade mistuning, Journal of Vibration, Acoustics, Stress, and Reliability in Design, 106, 204-210.

Huang, W.-H., 1982. Vibration of some structures with periodic random parameters, AIAA Journal, 20, 1001-1008.

Kaza, K.R.V. and Kielb, R.E., 1985. Vibration and flutter of mistuned bladed-disk assemblies, Journal of Propulsion, 1, 336-344.

Kielb, R.E. and Kaza, K.R.V., 1984. Effects of structural coupling on mistuned cascade flutter and response, Journal of Engineering for Gas Turbines and Power, 106, 17-24.

Lin, C.C. and Mignolet, M.P., 1997. An adaptive perturbation scheme for the analysis of mistuned bladed disks, Journal of Engineering for Gas Turbines and Power, 119, 153-160.

Mignolet, M.P. and Lin, C.C., 1993. The combined closed form - perturbation approach to the analysis of mistuned bladed disks, Journal of Turbomachinery, 115, 771-780.

Mignolet, M.P. and Lin, C.C., 1996. A novel limit distribution for the analysis of randomly mistuned bladed disks, ASME Paper 96-GT-41, Presented at the 41st International Gas Turbine and Aeroengine Congress and Exposition, United Kingdom.

Sinha, A., 1986. Calculating the statistics of forced response of a mistuned bladed disk assembly, AIAA Journal, 24, $1797-$ 1801.

Sinha, A. and Chen, S., 1989. A higher order technique to compute the statistics of forced response of a mistuned bladed disk, Journal of Sound and Vibration, 130, 207-221.

Valero, N.A. and Bendiksen, O.O., 1986. Vibration characteristics of mistuned shrouded blade assemblies, Journal of Engineering for Gas Turbines and Power, 108, 293-299.

Wei, S.-T. and Pierre, C., 1988. Localization phenomena in mistuned assemblies with cyclic symmetry - Part II: Forced vibrations, Journal of Vibration, Acoustics, Stress, and Reliability in Design, 110, 439-449.

Whitehead, D.S., 1966. Effect of mistuning on the vibration of turbomachines blades induced by wakes, Journal of Mechanical Engineering Science, 8, 15-21. 


\section{ait \\ ENERGY MATERIALS}

M A N E Y publishing

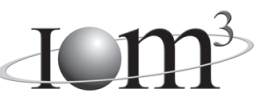

\section{Materials Science \& Engineering for Energy Systems}

Maney Publishing on behalf of the Institute of Materials, Minerals and Mining

The Institute of Materials, Minerals \& Mining

Economic and environmental factors are creating ever greater pressures for the efficient generation, transmission and use of energy. Materials developments are crucial to progress in all these areas: to innovation in design; to extending lifetime and maintenance intervals; and to successful operation in more demanding environments. Drawing together the broad community with interests in these areas, Energy Materials addresses materials needs in future energy generation, transmission, utilisation, conservation and storage. The journal covers thermal generation and gas turbines; renewable power (wind, wave, tidal, hydro, solar and geothermal); fuel cells (low and high temperature); materials issues relevant to biomass and biotechnology; nuclear power generation (fission and fusion); hydrogen generation and storage in the context of the 'hydrogen economy'; and the transmission and storage of the energy produced.

As well as publishing high-quality peer-reviewed research, Energy Materials promotes discussion of issues common to all sectors, through commissioned reviews and commentaries. The journal includes coverage of energy economics and policy, and broader social issues, since the political and legislative context influence research and investment decisions.

\section{CALL FOR PAPERS}

Contributions to the journal should be submitted online at http://ema.edmgr.com

To view the Notes for Contributors please visit: www.maney.co.uk/journals/notes/ema

Upon publication in 2006, this journal will be available via the Ingenta Connect journals service. To view free sample content online visit: www.ingentaconnect.com/content/maney

For further information please contact:

Maney Publishing UK

Tel: +44 (0)113 2497481 Fax: +44 (0)1132486983 Email: subscriptions@maney.co.uk

or

Maney Publishing North America

Tel (toll free): 8662975154 Fax: 6173546875 Email: maney@maneyusa.com

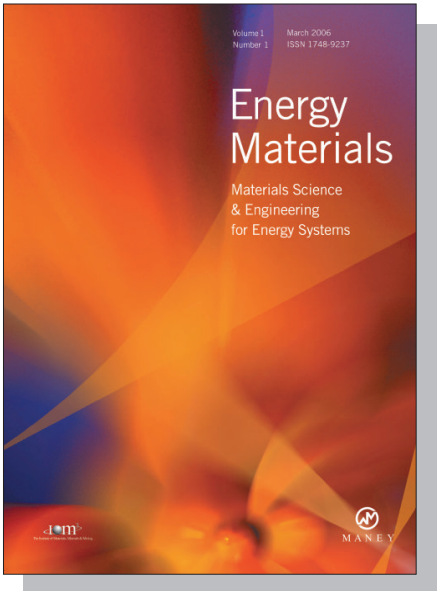

EDITORS

Dr Fujio Abe

NIMS, Japan

Dr John Hald, IPL-MPT, Technical University of Denmark, Denmark

Dr R Viswanathan, EPRI, USA

\section{SUBSCRIPTION INFORMATION}

Volume 1 (2006), 4 issues per year

Print ISSN: 1748-9237 Online ISSN: 1748-9245

Individual rate: $£ 76.00 / U S \$ 141.00$

Institutional rate: $£ 235.00 /$ US $\$ 435.00$

Online-only institutional rate: $£ 199.00 / U S \$ 367.00$

For special $\mathrm{IOM}^{3}$ member rates please email

subscriptions@maney.co.uk

\section{For further information or to subscribe online please visit www.maney.co.uk}



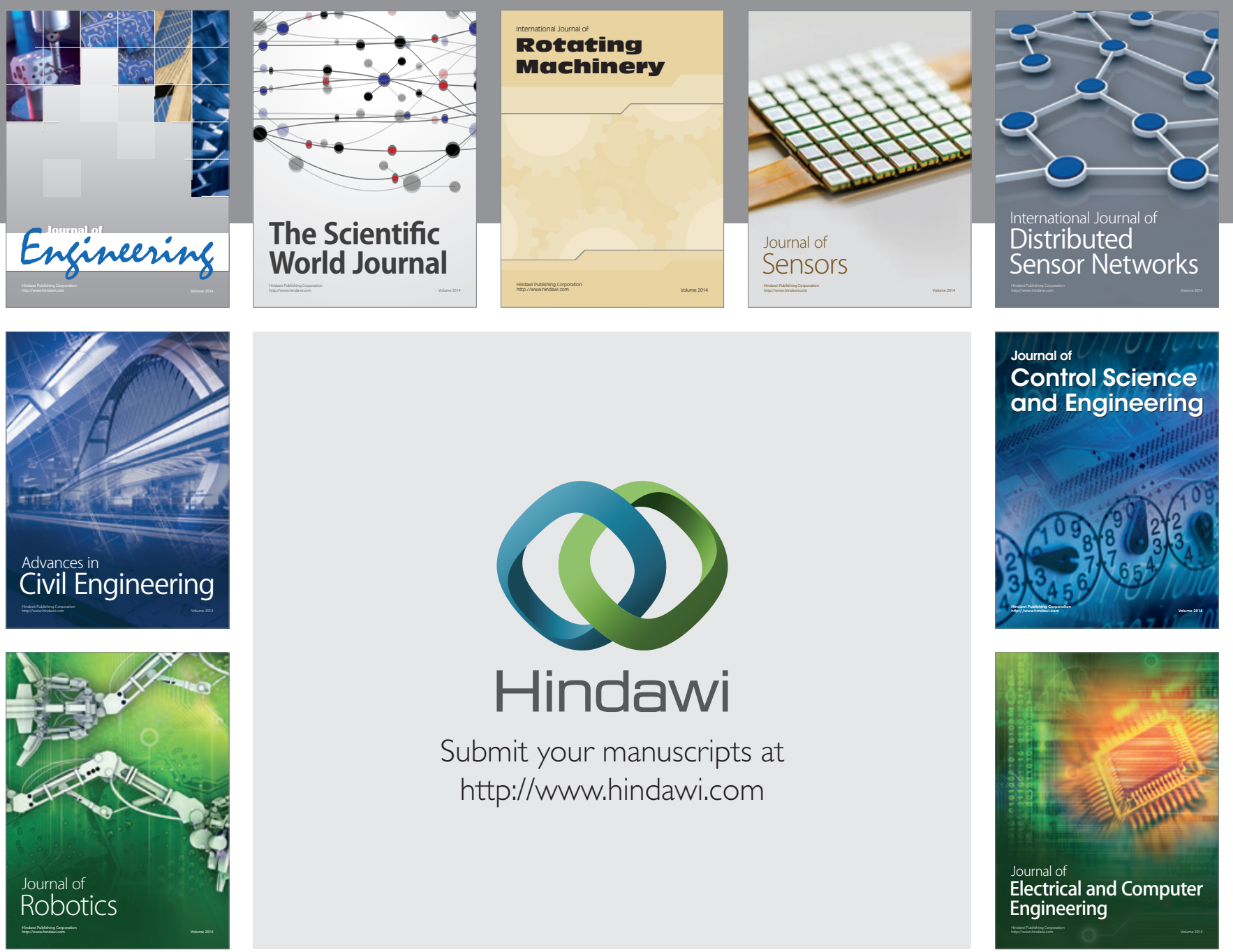

Submit your manuscripts at

http://www.hindawi.com
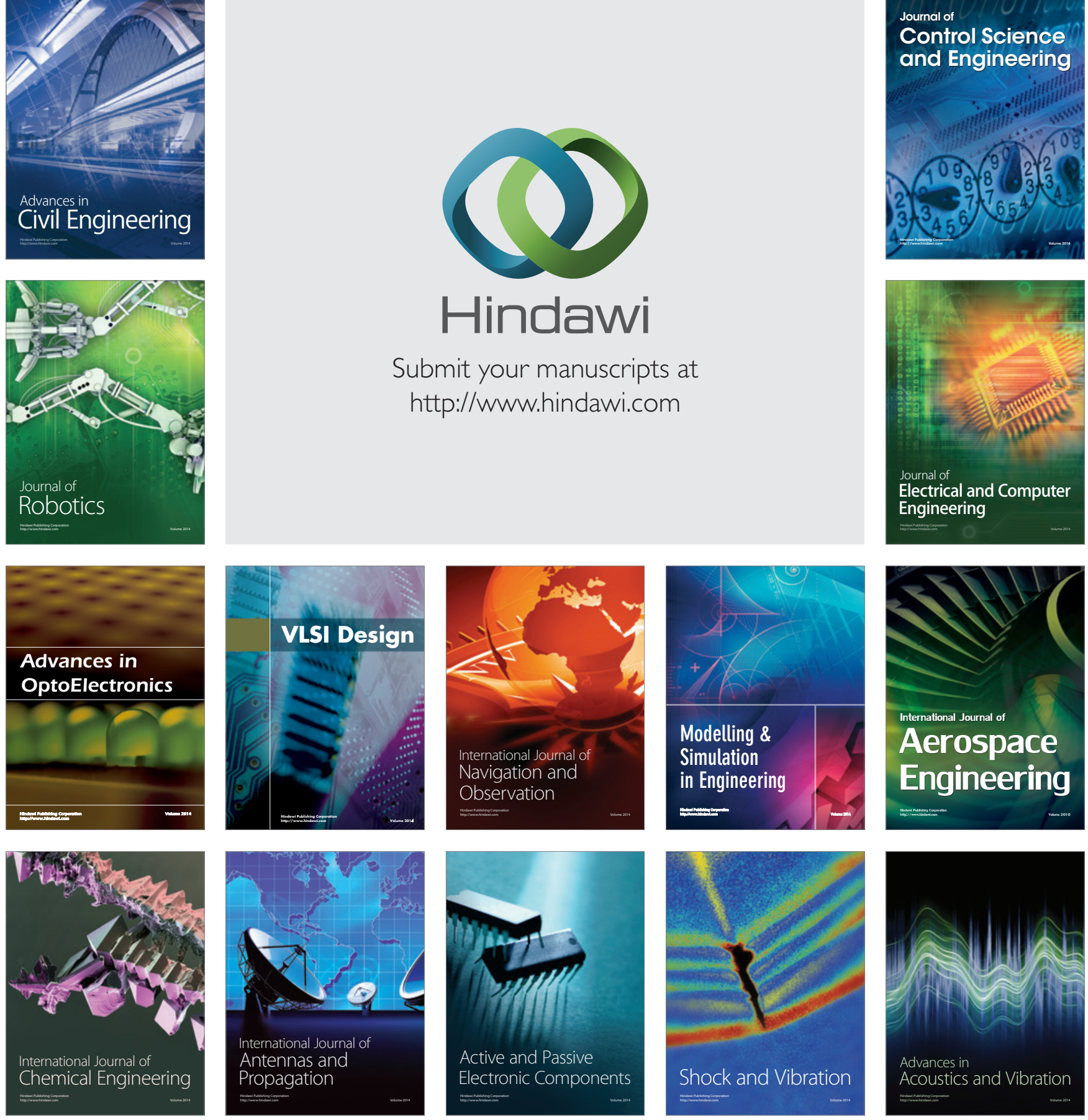